Textures and Microstructures, Vol. 31, pp. 151-175 Reprints available directly from the publisher Photocopying permitted by license only
(C) 1999 OPA (Overseas Publishers Association) N.V. Published by license under the Gordon and Breach Science Publishers imprint Printed in Malaysia.

\title{
NEW PARAMETER MODEL FOR TEXTURE DESCRIPTION IN STEEL SHEETS
}

\author{
L. DELANNAY*, P. VAN HOUTTE and A. VAN BAEL \\ Department MTM, Katholieke Universiteit Leuven, de Croylaan 2, \\ B-3001 Leuven, Belgium
}

(Received 31 July 1998)

\begin{abstract}
A new model is proposed for the characterisation of steel sheet textures. This model relies on the identification of 25 relevant parameters in the Orientation Distribution Function (ODF). Textures consisting of alpha- and gamma-fibres and/or cube and Goss components can be generated. The model is mathematically formulated and an automatic parameter identification technique is presented.

It was found that the model can quantitatively reproduce almost any industrial steel sheet texture. Based on this parameter model, a method is presented to systematically study the sensitivity of material properties on texture.
\end{abstract}

Keywords: Quantitative texture analysis; Model components; Steel sheet; Crystallographic fibres

\section{INTRODUCTION}

During the last decades, the Orientation Distribution Function (ODF) has been extensively used as quantitative description of materials' crystallographic texture. In spite of the accumulated experience on the ODF mathematical formulation and its applications, the visual interpretation and related physical characterisation of the ODF are still a concern for both academic and industrial researchers in the field of texture. The visual interpretation of an ODF is hindered by the fact that the ODF is defined in a three-dimensional, distorted "orientation" space (the Euler space), which is furthermore subjected to a number of complicated

\footnotetext{
* Corresponding author.
} 
symmetry rules. Hence, it is difficult to describe in simple terms the differences observed among textures measured on various materials. In order to investigate texture dependent properties of the material, it would thus be very helpful to define a reduced parameter set suitable for physical ODF description. The parameters should ideally allow the characterisation of any experimental texture of the given material. They should be accessible by a rigorous identification technique, and they should have a physical meaning in the sense that they improve threedimensional visualisation of the ODF.

The most appropriate and most widely spread mathematical expression of the ODF is the series expansion of generalised spherical harmonics, developed by Bunge (1982). The series expansion coefficients are however too numerous (several hundreds) to serve as practical parameter set for textures comparison. Moreover, they have no physical meaning which makes it impossible to select those coefficients that would be most relevant to describe or compare the textures. Note that if, on the other hand, the ODF is computed on a grid covering the orientation space, the number of values needed is even larger $(19 \times 19 \times 19$ points for a $5^{\circ}$ grid in orthorhombic-cubic symmetry). This number can be reduced by using a "nearly equal distant grid" (Helming, 1997) but, even then, more than 1000 points are necessary. Several attempts have been made to lower the number of parameters describing the ODF. Models consisting of a reduced set of parameters are of two types: they either involve computation of crystallographic volume fractions, or texture reproduction as a sum of model functions.

The computation of volume fractions or "weights" of typical crystallographic components is a very common technique to describe texture. A review of the different methods to obtain these weights is given by Cortie (1997). If one disposes of the ODF series expansion coefficients, computation of the crystallographic weights can most efficiently be carried out by making the convolution of the ODF with a gaussian distribution placed on the orientation of interest (see Bunge (1982) and Van Houtte (1995)). Procedures have been proposed to automatically identify the principal crystallographic components and compute their weight (see for example Cai and Lee (1994)). There are however two drawbacks to this kind of texture description. Firstly, such weights cannot conveniently be used to characterise the fibres that are observed, for example, in a steel sheet texture. Secondly, experimental ODFs often 
have peaks which do not exactly match with the precise orientation of the typical texture components. The components can be slightly shifted in all directions in orientation space.

The other way to efficiently characterise texture, is to make use of model functions. Examples of model functions are gaussian components (Bunge, 1982), complete or "partial" (Dnieprenko and Divinski, 1994) fibre components, "standard distributions" (Matthies et al., 1987), and elliptical components (Eschner, 1993; Helming et al., 1998). In all of these studies, a model texture is generated by superposing a set of components. Experimental textures are then characterised by fitting the model texture to the experimental one. This implies the determination of, for example, the intensity, position, scatter-width and direction of the different components. The value of these parameters is determined by least-squares fitting of the model ODF (Hirsch and Lücke, 1985), or model pole figures (Savyolova, 1993; Helming, 1998), to the experimental ones. It is reported that sets of 10-30 components provide a good reproduction of experimental textures. The total number of parameters is then 50-100.

The model presented in this paper is aimed to further reduce the number of parameters needed for the texture description by considering only one type of material, namely: steel sheets. The model makes use of model functions, but its parameters are directly related to visual features of the ODF: intensity, thickness, and position of the typical crystallographic fibres and components. Three-dimensional interpretation of the ODF is then improved. Moreover, since the parameters are not extracted from complicated mathematical formulas, the model is easy to use. A set of 25 parameters allows almost as detailed texture characterisation as other procedures relying on model functions. The parameters of the model are defined extensively in Section 1.

The first application of the model is the characterisation of experimentally observed textures. In order for such a characterisation to make sense, a systematic and objective technique must be available to identify the set of parameter values that corresponds to each investigated texture. Two Fortran routines have been written for this purpose. The first routine allows the generation of the series expansion of the texture corresponding to any given set of parameter values. The second routine makes use of this texture generation to find the parameter set that gives the best fit to the experimental texture to be studied. Section 2 contains a short description of the computer implementation of the model. 
The efficiency of these routines to reproduce all types of industrial steel sheet textures is discussed in Section 3. Four typical steel sheet textures have been reproduced. The reproduced ODF are quantitatively compared to the experimental ones, showing satisfactory match. Another application of the model is the study of the sensitivity of a steel sheet property on texture. A method is presented to use the model in such practical studies. The authors plan to apply this method to the study of steel sheet plastic planar anisotropy and formability in future contributions.

Final conclusions concerning the applicability and efficiency of the model are presented in Section 5.

\section{DESCRIPTION OF THE MODEL PARAMETERS}

Crystallographic textures in steel sheets generally consist of two fibres and a couple of individual components. The "alpha"-fibre contains orientations for which the $\langle 110\rangle$ crystal direction is aligned with the rolling direction $(\mathrm{RD})$; whereas orientations along the "gamma"-fibre are such that the $\langle 111\rangle$ direction is aligned with the normal direction (ND). Besides these fibres, the most frequent individual components are the "Goss": $\{110\}\langle 001\rangle$, and the "cube": $\{100\}\langle 001\rangle$ components. As already mentioned in the introduction, the exact position of these fibres and components varies from one texture to another. Components such as $\{554\}\langle 225\rangle$ can then be seen as belonging to a shifted gamma-fibre. The Goss component, on the other hand, is often shifted in the direction of the cube component along what one might call the "theta"-fibre $(\langle 001\rangle$ aligned with RD). For convenience, the model proposed here includes the "Goss" and "cube" components into this common "theta"-fibre. The model thus describes steel sheet textures as the superposition of three fibres: alpha, gamma and theta. All of these fibres are to be found in the $\varphi_{1}=0^{\circ}$ and $\varphi_{2}=45^{\circ}$ sections of the orientation space as illustrated in Fig. 1.

The model that is proposed is only applicable to textures having an orthorhombic-cubic symmetry which normally is the case in cold rolled steel sheets. Under this assumption, the texture can completely be described inside the portion of the orientation space in which the three Euler angles range from $0^{\circ}$ to $90^{\circ}$. This orientation subspace still 


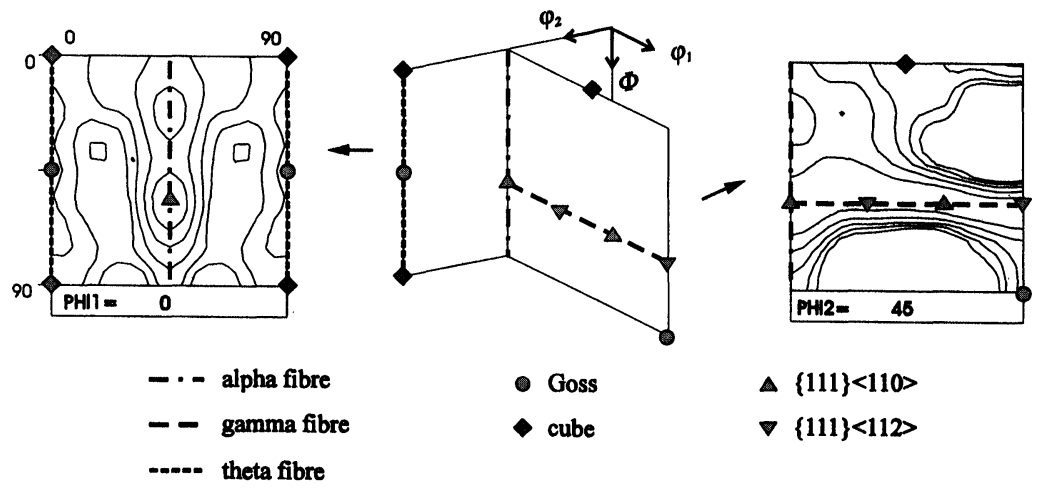

FIGURE $1 \varphi_{1}=0^{\circ}$ and $\varphi_{2}=45^{\circ}$ sections of the ODF, showing the principal fibres and components in a typical steel sheet texture (see also the 3 equivalent ranges along gamma).

contains several symmetrically equivalent representations of the texture components. The gamma-fibre, for example, can be split into three symmetrically equivalent segments: $\varphi_{1}=0-30^{\circ} ; \varphi_{1}=30-60^{\circ} ;$ and $\varphi_{1}=60-90^{\circ}$ (see Fig. 1). To restrict the number of parameters to a minimum and to avoid interferences between the parameter values, only one of the symmetrically equivalent representations of the fibre is characterised in the model: for example, the gamma-fibre is only described along $\varphi_{1}=0-30^{\circ}$.

There are surely many ways to define parameters suitable to describe the three fibres that we are dealing with. The parameter set that we have chosen, which proved to be convenient for the further applications, can best be presented in the three following steps, leading to a model of increasing complexity:

- The first step is the definition of the position of the three fibres in the ODF. The position of a fibre, also called skeleton line, is defined by searching the maximal (ODF) intensity in planes perpendicular to the fibre, lying at regular intervals along the fibre. By applying this to a number of experimental textures, we made the following observations. Firstly, the alpha- and theta-fibres most often lie at their ideal position, whereas the gamma-fibre is shifted. Secondly, one may consider, as a simplification, that the shifted gamma-fibre follows a straight line from $\varphi_{1}=0-30^{\circ}$. Thirdly, the position of the ODF peaks along the three fibres varies from one texture to the other. In order to 
restrict the number of parameters, only the orientation shifts that are needed to describe these features are included into the model (see below).

- The second step of our model elaboration consist of choosing, along the skeleton line of the fibres, a series of orientations at which the (ODF) intensity should be recorded. These orientations are chosen in such a way that the intensity evolves almost linearly from one orientation to the next, along the fibre axis (as illustrated on Fig. 5 in the next section). More precisely, we include as first parameters in our model the intensities measured at

- 5 orientations along alpha: $a_{1}$ to $a_{5}$,

- 3 orientations along gamma: $g_{1}\left(=a_{3}\right), g_{2}, g_{3}$,

- 3 orientations along theta: $t_{1}$ (=cube), $t_{2}, t_{3}$ (= Goss).

This makes a total of 10 points covering the three fibres that we aim to describe (as illustrated in Fig. 2). The orientation shifts that are allowed for each of these 10 points are given in Table I. It is supposed that the shifts vary linearly from one point to the next, similarly to the intensities. In other words, the model fibres are obtained by a kind of linear fitting of the experimental ones.

- The last step is to define the "thickness" of the fibre at each of the points described in step two. By "thickness", we understand the way the intensity decreases from the fibre central axis to distances away from it. To compute this thickness, we make use of the statistical concept of variance. This is justified by the fact that the (ODF) intensity represents the probability that a grain has a given crystal orientation. The thickness of a fibre can then be seen as the variance of
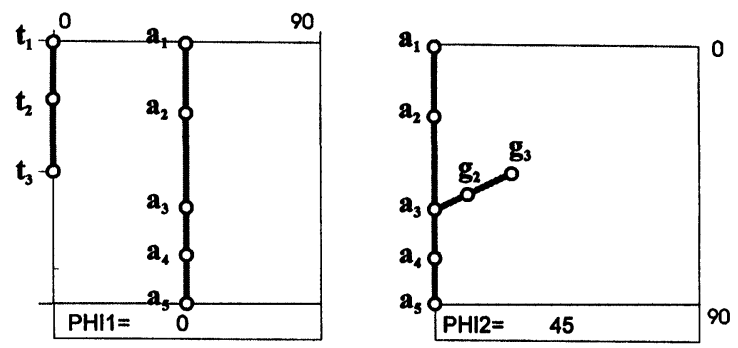

FIGURE 2 Points used by the model to describe the fibres. 
TABLE I Summary of the model parameters

\begin{tabular}{lccccccc}
\hline Name & $\begin{array}{c}\text { Ideal position } \\
\left(\varphi_{1}, \Phi, \varphi_{2}\right)\end{array}$ & Intensity & $\begin{array}{c}\text { Thickness } \\
\text { along } \varphi_{2}\end{array}$ & $\begin{array}{c}\text { Thickness } \\
\text { along } \varphi_{1}\end{array}$ & $\begin{array}{c}\text { Shift } \\
\text { along } \varphi_{1}\end{array}$ & $\begin{array}{c}\text { Shift } \\
\text { along } \Phi\end{array}$ & $\begin{array}{c}\text { Shift } \\
\text { along } \varphi_{2}\end{array}$ \\
\hline$a_{1}$ & $0,0,45$ & $P_{1}$ & $P_{10}$ & & & & \\
$a_{2}$ & $0,30,45$ & $P_{2}$ & $P_{11}$ & $P_{18}$ & & $P_{20}$ & \\
$a_{3}=g_{1}$ & $0,55,45$ & $P_{3}$ & $P_{12}$ & & & $P_{21}$ & \\
$a_{4}$ & $0,70,45$ & $*$ & $*$ & & & $P_{22}$ & \\
$a_{5}$ & $0,90,45$ & $P_{4}$ & $P_{13}$ & & $P_{19}$ & & \\
$g_{2}$ & $15,55,45$ & $P_{5}$ & $P_{14}$ & & & $P_{23}$ & $P_{25}$ \\
$g_{3}$ & $30,55,45$ & $P_{6}$ & $P_{15}$ & & & & \\
$t_{1}$ & $0,0,0$ & $P_{7}$ & + & & & $P_{24}$ & \\
$t_{2}$ & $0,25,0$ & $P_{8}$ & $P_{16}$ & & & & \\
$t_{3}$ & $0,45,0$ & $P_{9}$ & $P_{17}$ & & & & \\
\hline
\end{tabular}

${ }^{*}$ No specific parameter is defined although the intensity/thickness value is imposed. ${ }^{\dagger}$ The thickness is left free at this point.

the intensity around the axis of the fibre. This variance is computed by measuring the intensity at different orientations in a section perpendicular to the fibre axis. We call these orientations "satellites". Figure 3 shows the position of the satellites in a section of the gammafibre. Our experience has taught us that, for most of the orientations of step two, we could best measure the thickness along a unique direction of the orientation space (the $\varphi_{2}$ direction). For the point $\mathrm{a}_{2}$, however, a second thickness should be defined (along $\varphi_{1}$ ). The thickness $V_{i}$ at a given orientation $i$ is defined as:

$$
V_{i}=\frac{\sum_{\mathrm{sat}=1,4} d_{\mathrm{sat}}^{2} I_{\mathrm{sat}}}{I_{i}+\sum_{\mathrm{sat}=1,4} I_{\mathrm{sat}}}
$$

where $I_{i}$ is the intensity at point $i$ along the fibre, and the $I_{\text {sat }}(\mathrm{sat}=1,4)$ are the intensities of the satellites that are measured at distances $d_{\text {sat }}$ equal to $5^{\circ}, 10^{\circ}, 15^{\circ}$ and $20^{\circ}$ from point $i$, in the direction of interest $\left(\varphi_{2}\right.$ or $\left.\varphi_{1}\right)$. It is supposed that the thickness varies linearly along the fibre; similarly to the intensity and the orientation shifts.

The 25 parameters defined in the model are summarised in Table I. The number of parameters has been reduced as much as possible by not considering shifts at places where experience has revealed that they do not bring much improvement to the model. For the same reason the intensity at $a_{4}$ is maintained equal to one, whereas its thickness is univocally determined by the parameters $\mathrm{P}_{4}$ and $\mathrm{P}_{13}$. (The only parameter proper to $a_{4}$ is $\mathrm{P}_{22}$ which defines the position of the point along $\Phi$.) 

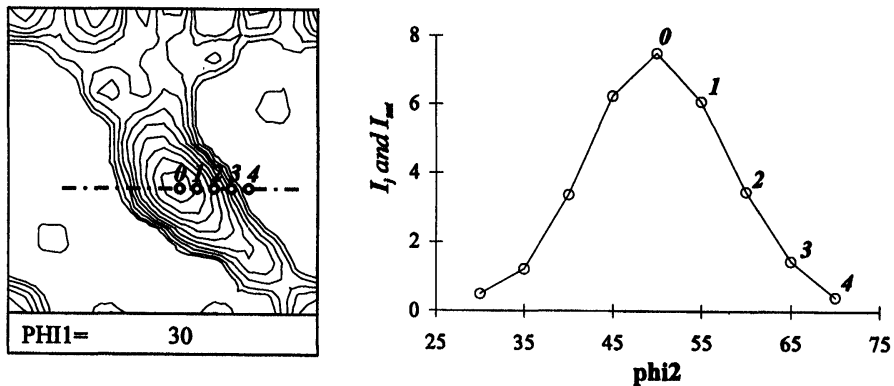

FIGURE 3 Points used for the computation of the thickness (see Eq. (1)). 0 stands for the centre of the fibre where $I_{i}$ is measured, and 1-4 stand for the 4 "satellites" (section of the gamma-fibre).

The thickness at $t_{1}$ (cube orientation) is left free. Note also that, as shown by Fig. $2, g_{2}$ is a point on the straight line connecting $a_{3}$ and $g_{3}$. This means that the parameters $P_{21}, P_{23}$ and $P_{25}$ also affect the position of $g_{2}$.

A characteristic of such a texture description model is that the knowledge of the parameter values does not directly provide the series expansion of the ODF. The parameters facilitate the three-dimensional visualisation of the ODF. We call this a "model of Type 1". It represents requirements for the ODF. In order to generate the series expansion of the model texture that would satisfy the latter requirements, we make use of a "model of Type 2": the model texture is generated as a sum of $N$ gaussian distributions (see Fig. 4). The parameters of this model of Type 2 are the positions, the maxima, and the spreads of the $N$ gaussians. Once they are known, the series expansion of the model texture can be computed (see below).

The model (of Type 1) described in this section can only be used in practice if we define a procedure to

- systematically determine the positions, maxima and spreads of the gaussians (parameters of Type 2) that lead to a generated texture which meets the requirements fixed by the 25 parameters (of Type 1);

- find the 25 parameter values that best reproduce any existing experimental texture.

Two Fortran routines have been written for these purposes. They are presented in the next section. In the following, when the term "parameters" is used without specification, it refers to the 25 parameters of Type 1 . 
a) Model of Type 1

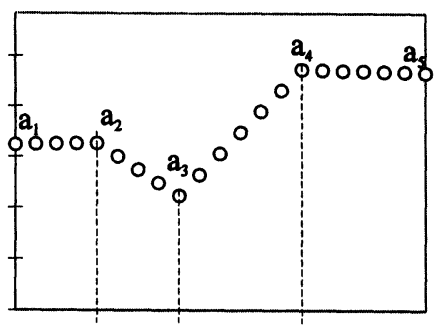

b) Model of Type 2 (sum of gaussians)

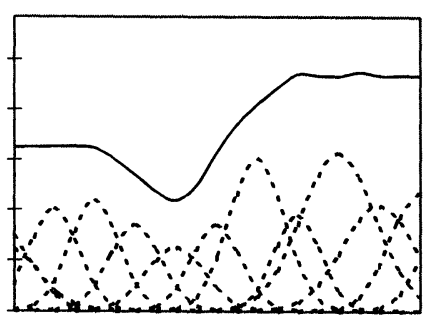

c) Fitting of the two models

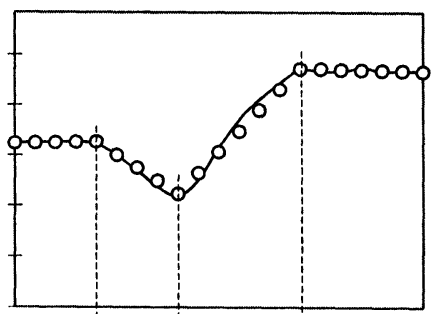

FIGURE 4 Generation of a model texture: (a) requirements imposed by the 25 parameters (Type 1); (b) model texture generated as a sum of gaussians (Type 2); (c) verification that the generated texture meets the requirements of the model of Type 1.

\section{COMPUTER IMPLEMENTATION OF THE MODEL}

Before we come to the description of the two routines that were developed, we should say a brief word about the ODF mathematical tools that are available to us. The ODFs that we investigate are expressed in the form of series of generalised spherical harmonic functions (see Bunge (1982)). The ODF is formulated as

$$
f(g)=\sum_{l=1}^{l_{\max }} \sum_{\mu=1}^{M(l)} \sum_{\nu=1}^{N(l)} C_{l}^{\mu \nu} \dot{\mathrm{T}}_{l}^{\mu \nu}(g) .
$$


In this expression, $g$ represents the crystal orientation, the $\dot{\mathrm{T}}_{l}^{\mu \nu}(g)$ are spherical harmonic functions that only depend on $g$, and the $C_{l}^{\mu \nu}$ are the coefficients of the series expansion that differ from one texture to the other. The three dots on $\ddot{\mathrm{T}}_{l}^{\mu \nu}(g)$ mean that both the sample and crystal symmetry are included in this term. For a particular experimental texture, the $C_{l}^{\mu \nu}$ coefficients of even rank are obtained by inversion of 4 incomplete pole figures measured by $\mathrm{X}$-ray diffraction, whereas the odd rank coefficients result of the ghost correction (Van Houtte, 1991; 1995). The series expansions that we use are truncated at $l_{\max }=32$. This leads to 524 different coefficients in the case of cubic crystal symmetry and orthorhombic sample symmetry.

Besides the symmetry, the normality of the ODF is another physical property which should be satisfied by any texture generated by the model. The ODF normality implies that

$$
\oint f(g) \mathrm{d} g=1 .
$$

The texture generated in our model is obtained by making the superposition of a set of spherical gaussian distributions. Note that they will not exclusively be placed on the positions $a_{1}, \ldots, a_{5}, g_{2}, g_{3}, t_{1}, t_{2}, t_{3}$. Other positions will also be used, as explained in Section 2.1. This procedure is motivated by the fact that experimentally observed texture components often have a shape which is close to a gaussian. Moreover, the use of gaussians is convenient for texture generation: on the one hand, the normality and the symmetry properties of the generated texture can easily be imposed; on the other hand, a simple analytical expression is available to compute the series expansion coefficients (see below).

The (ODF) intensity at a given point $i$, in the presence of $N$ gaussian components, is usually formulated as follows

$$
I_{i}=\sum_{k=1, N} S_{k} \exp \left(-\left(\phi_{i k} / \Phi_{k}\right)^{2}\right)
$$

where $I_{i}$ symbolises the intensity (equivalent to $\left.f\left(g_{i}\right) !\right), S_{k}$ is the maximum of the $k$ th gaussian, $\Phi_{k}$ is its scatter-width, and $\phi_{i k}$ is the angular distance between the point $i$ of interest and the $k$ th gaussian. So far the symmetry and normality of the ODF are not taken into account.

The best way to enforce sample and crystal symmetry in such a texture is the following: one should not only put gaussians at the $N$ orientations 
mentioned in (4), but also at all symmetrical redundancies of these orientations. In the case of orthorhombic-cubic symmetry, orientations have up to 96 equivalent redundancies (see for example Matthies et al. (1987)). This number is reduced in the case of "low multiplicity" orientations for which several redundancies coincide at the same orientation. If the texture is constructed by superposing the $N \times 96$ "symmetrical" gaussians (even for low multiplicity components), it is ensured that the symmetry rules are fulfilled. Equation (4) should therefore be replaced by

$$
I_{i}=\sum_{k=1, N} S_{k} \sum_{m=1,96} \exp \left(-\left(\phi_{i k}^{m} / \Phi_{k}\right)^{2}\right)
$$

where $\phi_{i k}^{m}$ is the angular distance between the point $i$ where the intensity is measured and the $m$ th symmetrical redundancy of the $k$ th gaussian.

The determination of redundant orientations can efficiently be carried out by making use of quaternions (Altmann, 1986). The way to proceed is to rotate the original orientation into its symmetrical equivalents. The following symmetry elements should be used. Three rotation axes in the cubic crystal lattice: $\langle 111\rangle$ (3-fold), $\langle 110\rangle$ (2-fold), and $\langle 100\rangle$ (4-fold); and two rotation axes in the sample reference system: RD (2-fold) and TD (2-fold). (The 2-fold rotation around ND is obtained by combining the two others.) This leads to $3 \times 2 \times 4 \times 2 \times 2=96$ equivalent ways to define the (right-handed!) crystal and sample reference systems, thus 96 equivalent sets of Euler angles. Quaternions are very convenient to perform such rotations. Furthermore they allow fast computation of angular distances (see also Van Acker (1996)).

The second important property of the texture is its normality as expressed by Eq. (3). When a texture is generated by a superposition of $N \times 96$ "symmetrical" gaussians, the normality is ensured by setting

$$
\text { Norm }=\frac{96}{\sqrt{4 \pi}} \sum_{k=1, N} S_{k} \Phi_{k}\left[1-\exp \left(-\frac{\Phi_{k}^{2}}{4}\right)\right]=1 .
$$

The coefficients of the series expansion corresponding to this same texture, are given by

$$
C_{l}^{\mu \nu}=\frac{96}{\sqrt{4 \pi}} \sum_{k=1, N} S_{k} \Phi_{k}\left[\exp \left(-\frac{P^{2} \Phi_{k}^{2}}{4}\right)-\exp \left(-\frac{(l+1)^{2} \Phi_{k}^{2}}{4}\right)\right] \ddot{T}_{l}^{* \mu \nu}\left(g_{k}\right),
$$


where $\ddot{\mathrm{T}}_{l}^{* \mu \nu}$ is the complex conjugate of the harmonic function. Note that, in the case of orthorhombic-cubic symmetry, $\ddot{\mathrm{T}}_{l}^{\mu \nu}$ has no imaginary part, and the two functions are equal. The reader should refer to Bunge (1982) for the derivation of these expressions.

\subsection{Generation of the Texture Corresponding to a Given Set of Parameters}

In the context of our model, the generated texture must have characteristics that are defined by the parameter values. This means that the (linear) evolution of the intensity, thickness, and orientation shifts are imposed along the fibres of the generated ODF.

Our experience has shown that such a texture could be obtained by making use of 35 gaussians. The latter gaussians are placed at approximately equal distance of one another along the three shifted fibres. The position of the gaussians is thus univocally determined by the orientation shift parameter values. The maximum $S_{k}$ and the scatterwidth $\Phi_{k}$ of the gaussians, on the other hand, are obtained by imposing the intensity and thickness at different positions along the fibres. The intensities and thickness values to be imposed are fixed by the 25 parameter values and by the fact that their evolution is linear along segments of the fibres. The determination of $S_{k}$ and $\Phi_{k}$ for $k=1,35$ then implies that the following set of non-linear equations be solved:

$$
\begin{cases}I_{i}\left(S_{k}, \Phi_{k}\right)=I \operatorname{Limp}_{i}, & i=1, n_{I}, \\ V_{i}\left(S_{k}, \Phi_{k}\right)=\frac{\sum d_{\text {sat }}^{2} I_{\text {sat }}}{I_{i}+\sum I_{\text {sat }}}=V-\operatorname{imp}_{i}, & i=1, n_{V}, \\ \operatorname{Norm}\left(S_{k}, \Phi_{k}\right)=1 & \end{cases}
$$

where $I \_\mathrm{imp}_{i}$ and $V \mathrm{imp}_{i}$ are the imposed values of the intensity and thickness at point $i$. The intensities $I_{i}$ and $I_{\text {sat }}$ may be computed in two different ways, from the unknowns $S_{k}$ and $\Phi_{k}$.

The first way to compute the intensities relies on the series expansion (2) with the $C_{l}^{\mu \nu}$ coefficients given by (7). The values of the harmonic functions $\ddot{\mathrm{T}}_{l}^{\mu \nu}$ are computed beforehand at all orientations of interest, by making use of the library program proposed by Wagner (1985). The series is truncated at $l_{\max }=32$.

The second way to compute the intensities is the application of (5). The angular distances $\phi_{i k}^{m}$ are evaluated beforehand. According to (5), 
the intensity at $i$ is obtained by making the sum of a large number of exponential terms $(35 \times 96=3360)$. The contribution of "very" distant gaussians is however negligible. The computational work can therefore substantially be reduced by considering that each gaussian only contributes to the intensities of its closest neighbours.

Both techniques of intensity computation have been implemented in Fortran routines. It turned out that the routine making use of series expansions was twice as fast as the other one. Hence, it should be preferred for further work. Comparison of the two routines yielded another interesting result: the effect of the series expansion truncation could be estimated by comparing the truncated intensities to their real value, the latter being provided by the second routine (taking account of all exponential terms, this time). As expected, sharper gaussians lead to a more severe discrepancy between truncated and real intensities. In order to limit the detrimental effect of the series truncation, it was decided to impose to all gaussians a minimum scatter-width of $6^{\circ}$.

The unknowns of the equation set (8) are submitted to a number of constraints: the scatter-widths $\Phi_{k}$ must be greater or equal to $6^{\circ}$; and, for physical reasons, the maxima $S_{k}$ must be positive. Because of these constraints, we cannot find the value of the unknowns by making use of a classical algorithm for non-linear equation sets. We rather have to minimise a sum of squares composed of the equation residues and of penalty functions that tend to enforce the constraints. This least-squares minimisation is performed by making use of the Broyden-FletcherGoldfarb-Shanno (BFGS) algorithm as implemented in the Fortran routines package by Press et al. (1992).

One texture generation takes about $1 \mathrm{~min} 30 \mathrm{~s}$ on a Pentium II $233 \mathrm{MHz}$ with MMX processor. The stability of the algorithm has been checked by varying the starting point of the minimisation and by comparing solutions obtained using the two different intensity computation routines. Small fluctuations were observed on the final $S_{k}$ and $\Phi_{k}$ which have negligible effect on the further application of the routine.

\subsection{Determination of the Parameter Set Corresponding to a Given Experimental Texture}

The purpose of this routine is to identify the parameter set that must be attributed to a given experimental texture to allow its characterisation. 
The experimental texture is provided in the form of a series expansion as described above.

The problem is solved by making a first close approximation of the parameter values and then, iteratively, by generating a series of textures $f_{P_{1}, \ldots, P_{25}}(g)$ corresponding to different sets of parameter values, and by ultimately selecting the generated texture which is "closest" to the experimental texture. The whole identification procedure requires very little interaction with the user.

An initial estimation of the parameter values is easily obtained considering that all parameters are directly related to some (ODF) intensities. Since we dispose of the series expansion coefficients, the intensity can be computed for any orientation in the experimental texture. The procedure leading to the first estimation of the parameters is the following:

- We first determine the position of the gamma-fibre skeleton line by searching the maximal intensities in the sections $\varphi_{1}=0^{\circ}, 5^{\circ}, \ldots, 30^{\circ}$ of the experimental ODF. Note that the position of the two other fibres is fixed.

- We approximate the gamma skeleton line by a straight line.

- Using the series expansion coefficients of the experimental ODF, we compute the intensity and thickness at $5^{\circ}$ intervals along the three linear fibres.

- We deduce the orientation of the predominant peaks along each of the fibres. Once this is done, the orientations of the 10 points defined in the second step of our model elaboration (Section 2) are known.

- According to the model, the evolution of the intensity and thickness should be linear from one of the 10 points to the next. In the experimental fibre, this is of course not exactly true. It can be verified by comparing the intensity and thickness that have just been computed along the fibres to the linearly interpolated values.

- The "first" close estimation of the parameter values is then found iteratively as the set of values that leads to the best linear fit of the intensity and thickness evolutions along the fibres of the experimental ODF. The convergence is very fast ( $30 \mathrm{~s}$ are needed to obtain this first parameter set on the Pentium PC mentioned above).

To illustrate the first step of the identification procedure, we present in Fig. 5 the resulting linear fit of the intensity evolution along the alpha 

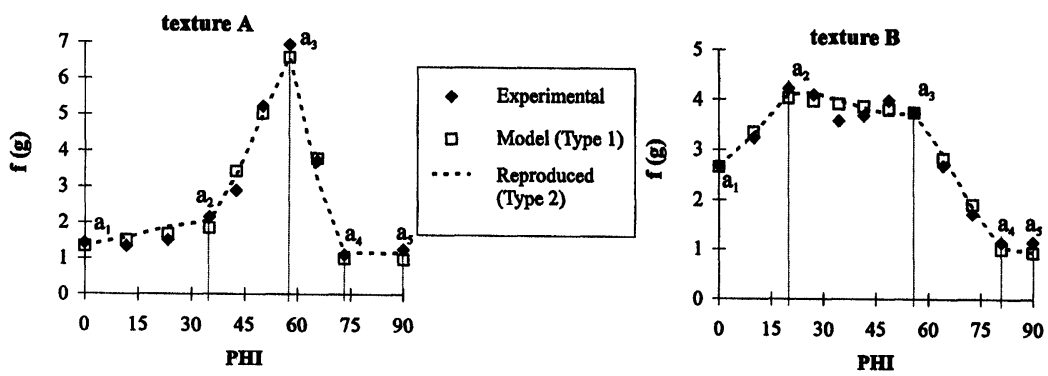

FIGURE 5 Linear fit of the intensity evolution along the alpha fibre of two experimental textures: (i) experimentally measured intensity; (ii) intensity imposed by the model parameters (Type 1); (iii) reproduced intensity obtained by application of routine $n^{\circ} 1$ (model of Type 2).

fibre of two different experimental ODFs. One fibre is somewhat better reproduced than the other. In both cases, however, this only constitutes a first approximation of the parameter values. Indeed, this parameter set only relies on the intensity and thickness along the skeleton line of the fibres, not on the complete ODF. We are probably close to the best reproduction of the ODF, but an improvement is possible by also accounting for intensities outside the fibres. This will be done in a second step.

During the second step of the parameter identification procedure, one tends to iteratively minimise the following expression:

$$
\operatorname{Min}_{P_{1}, \ldots, P_{25}}\left(\frac{\oint_{g}\left[f_{P_{1}}, \ldots, P_{25}(g)-f_{\exp }(g)\right]^{2} \mathrm{~d} g}{\oint_{g} f_{\exp }(g)^{2} \mathrm{~d} g}\right) .
$$

This expression is commonly used to quantitatively distinguish textures that are close to each other (see for example Hirsch and Lücke (1985) and Schouwenaars and Van Houtte (1994)). It yields a low value only if the experimental and model textures are very similar over the whole orientation space.

Expression (9) can be simplified by making use of the harmonic series. We have:

$$
\oint_{g} f(g)^{2} \mathrm{~d} g=\sum_{l} \frac{1}{1+2 l} \sum_{\mu} \sum_{\nu} C_{l}^{\mu \nu^{2}} .
$$


Expression (9) can thus be replaced by:

$$
\operatorname{Min}_{P_{1}, \ldots, P_{25}}\left(\frac{\sum_{l} \frac{1}{1+2 l} \sum_{\mu} \sum_{\nu}\left(C_{l P_{1}, \ldots, P_{25}}^{\mu \nu}-C_{l \exp }^{\mu \nu}\right)^{2}}{\sum_{l} \frac{1}{1+2 l} \sum_{\mu} \sum_{\nu} C_{l \exp }^{\mu \nu^{2}}}\right) .
$$

Since the series expansion coefficients of the experimental texture are known and those of the generated texture can be obtained by applying the first routine, we are able to evaluate the latter expression.

We are then again confronted with a non-linear minimisation problem. The solution of the problem is however hindered by the fact that we do not dispose of an analytical expression for the function to be minimised, nor for its gradient. This is a serious inconvenience when performing such minimisation. We make use of the algorithm of Powell as implemented in the Fortran routines package by Press et al. (1992) which has the drawback to require a large number of function evaluations (each of them implying an application of the first routine!).

Because the parameters have a physical significance and because they should not interfere with each other, we expect to converge to a unique solution if the starting point of the iterative procedure is close enough to the final solution. The application of the routine is quite lengthy since a lot of function evaluations are needed and each of them requires $1 \mathrm{~min} 30 \mathrm{~s}$. In total, the parameter values identification takes about $10 \mathrm{~h}$, on the computer already mentioned.

Note, however, that we need this procedure only to derive the parameter description of an already known texture, so that we can assess the difference between the original texture and the one calculated from the model. The procedure is not needed in applications such as studies of the sensitivity of certain texture dependent properties to the parameters of the model (see below).

\section{RESULTS AND DISCUSSION}

\subsection{Reproduction of Experimental Textures}

The parameter model that is described has been tested on a large number of experimental steel sheet textures. Four of them are selected here to 
illustrate the capabilities of the model. They are labelled texture A, B, $\mathrm{C}$ and D. Each of them represents an industrial ULC- or IF-steel after cold rolling and sometimes annealing. The ODF of these textures were deduced from the experimentally measured pole figures (as explained in Section 2). The complete ODF of texture A and the $\varphi_{1}=0^{\circ}$ and $\varphi_{2}=45^{\circ}$ ODF sections of textures B, C and D are given in Figs. 6-9.

The latter figures also contain the ODF sections of the textures reproduced by the model. As explained in the previous section, the fitting of the model parameters is based on the minimisation of expression (9). The final values obtained for these minimisations are given in Table II for all 4 textures. (The meaning of the other values in this table is explained below.)

The entire ODF of texture $A$ is presented to show the good match between the experimental and the model texture: not only the $\varphi_{1}=0^{\circ}$ and $\varphi_{2}=45^{\circ}$ sections containing the 3 fibres are well reproduced. This was also true for textures $\mathrm{B}, \mathrm{C}$ and $\mathrm{D}$. To check that no other important component was omitted, we have computed the difference between the generated ODFs and the experimental ones, on a $5^{\circ}$-grid covering the orientation subspace. The mean and maximal absolute values of this difference and the orientation were the maximum occurs are also given in Table II. The values obtained for these differences may seem important compared to the mean value of $f(g)$ which of course is 1 . It is however more relevant to compare them to the maximum intensity measured in the ODF (which is also given in Table II). The averaged and maximum absolute differences are respectively around $5 \%$ and $25 \%$ of the maximal ODF intensity. These discrepancies are due to the fact that the model does not account for minor isolated peaks outside the fibres, and that perpendicular sections of the fibres do not have exact gaussian distributions. Such discrepancies could be reduced by increasing the number of parameters. However, we prefer to keep our reduced number of parameters since this is most convenient for the future applications of the model, as shown in the following.

In the next section, we illustrate how the model can be used to study the sensitivity of a steel sheet property on texture. The properties that can be studied are those for which a reliable prediction software is available. To start such a study, it is required that the predicted value of the property be very similar for the experimental and the reproduced texture. In other words, the discrepancies mentioned in Table II should 


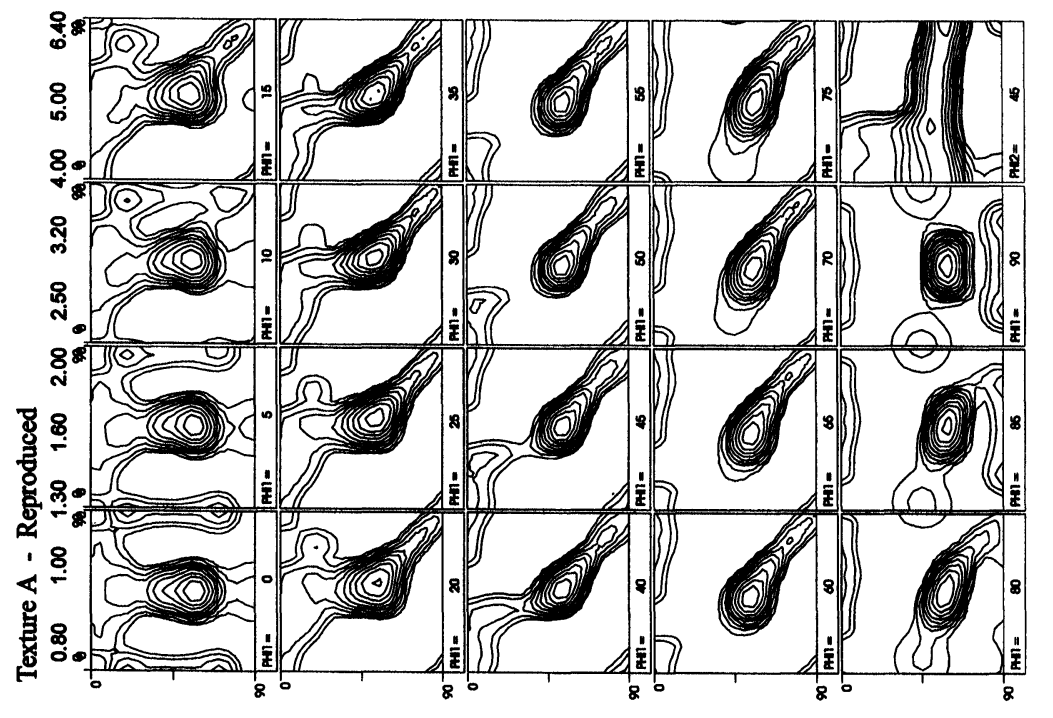

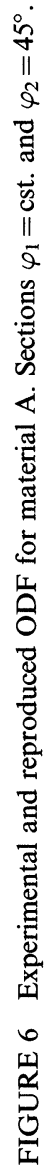

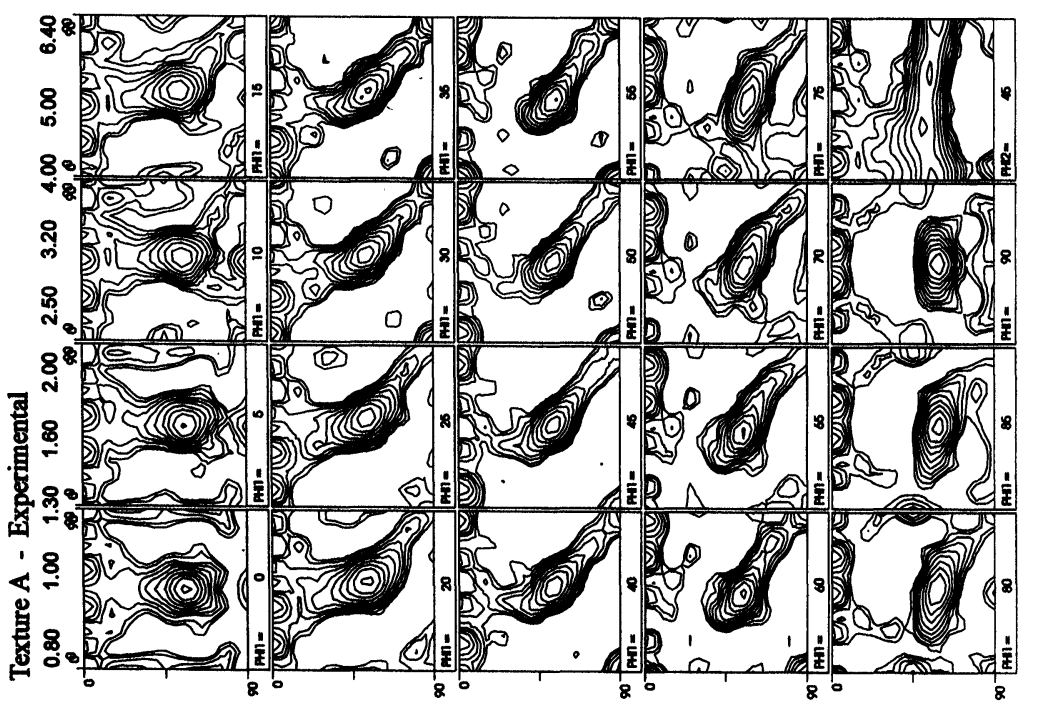



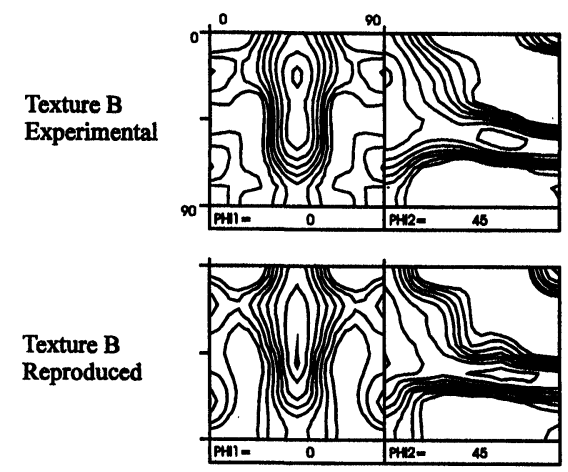

FIGURE 7 Experimental and reproduced ODF for material B. Sections $\varphi_{1}=0^{\circ}$ and $\varphi_{2}=45^{\circ}$ (same contour lines as Fig. 6).

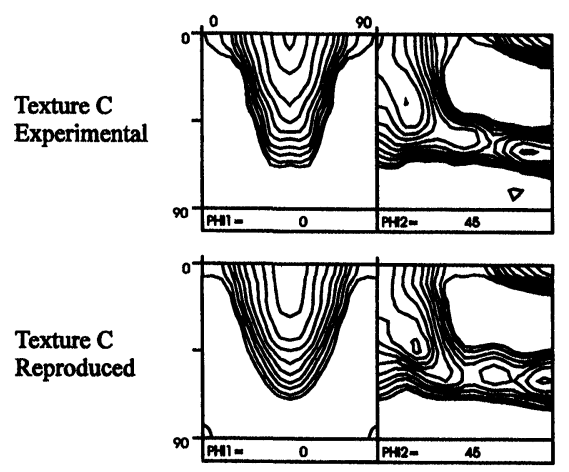

FIGURE 8 Experimental and reproduced ODF for material C. Sections $\varphi_{1}=0^{\circ}$ and $\varphi_{2}=45^{\circ}$ (same contour lines as Fig. 6).

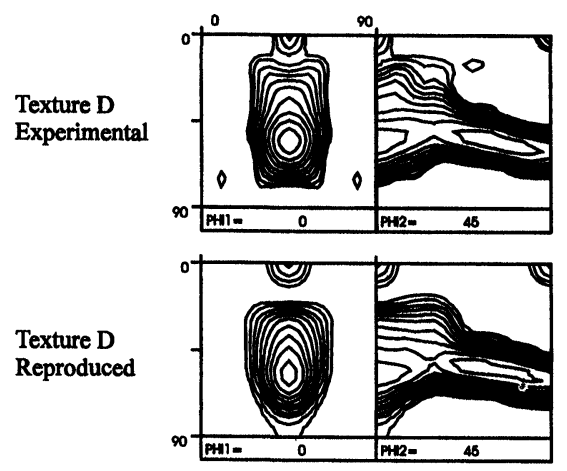

FIGURE 9 Experimental and reproduced ODF for material D. Sections $\varphi_{1}=0^{\circ}$ and $\varphi_{2}=45^{\circ}$ (same contour lines as Fig. 6). 
TABLE II Summary of the experimental texture reproductions

\begin{tabular}{lccccc}
\hline $\begin{array}{l}\text { Texture } \\
\text { label }\end{array}$ & $\begin{array}{c}\text { Minimal value } \\
\text { for expression (9) }\end{array}$ & $\begin{array}{c}\text { Maximal } \\
\text { ODF-intensity* }\end{array}$ & $\begin{array}{c}\text { Averaged } \\
\text { absolute } \\
\text { difference* }\end{array}$ & $\begin{array}{c}\text { Maximum } \\
\text { absolute } \\
\text { difference* }\end{array}$ & $\begin{array}{c}\text { Position of } \\
\text { maximum absolute } \\
\text { difference* }\left(\varphi_{1}, \Phi, \varphi_{2}\right)\end{array}$ \\
\hline A & 0.053 & 7.91 & 0.30 & 1.66 & $35,0,0$ \\
B & 0.031 & 4.23 & 0.19 & 0.89 & $50,45,40$ \\
C & 0.044 & 6.85 & 0.24 & 1.88 & $75,60,45$ \\
D & 0.032 & 7.76 & 0.21 & 1.42 & $45,40,35$ \\
\hline
\end{tabular}

* calculated in a $5^{\circ}$-grid covering the Euler space.
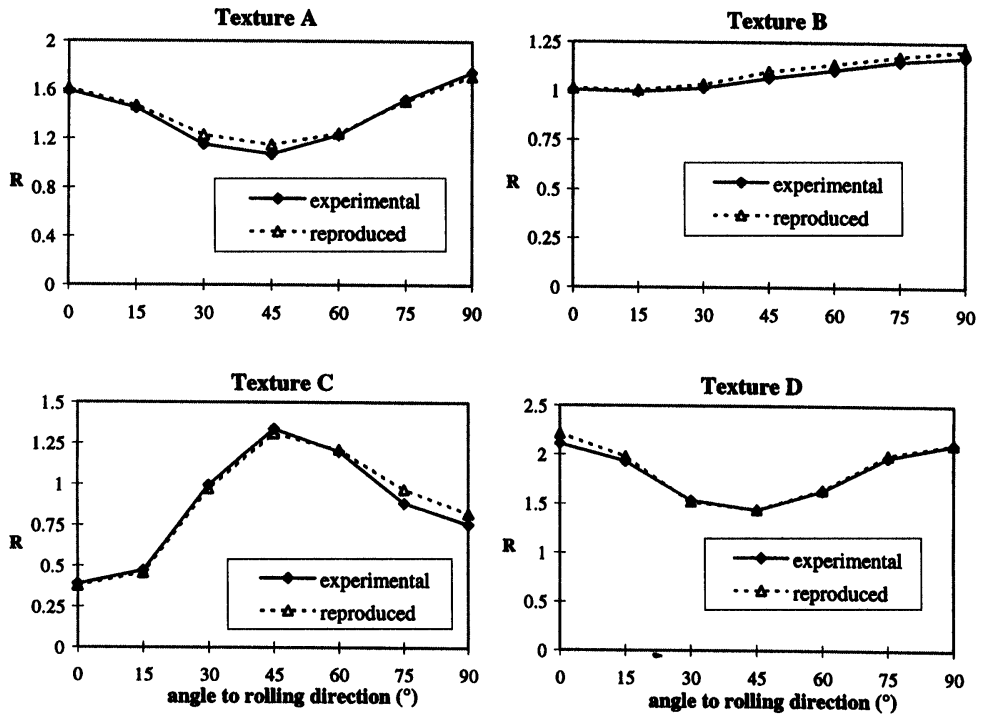

FIGURE 10 Comparison of the $R$-value profiles predicted from the experimental and reproduced textures.

have minor influence on the value of the property as calculated by our software. As an example, we have tested this for the case of planar anisotropy, which is characterised by the $R$-value profile as a function of the azimuthal direction in the plane of the sheet. The $R$-value can be predicted by making use of the full-constraints Taylor model (see Schouwenaars et al., 1994; 1996). The R-value profiles that we have obtained for the experimental and reproduced textures are given in Fig. 10. They correspond remarkably well although the texture reproduction was strictly based on the minimisation of (9). This means 
that the reproduced textures presented in Figs. 6-9 could serve as basis of a sensitivity study of planar anisotropy on texture.

We should also mention that some textures could not be well reproduced by the model. Those are either almost random hot rolled textures, or, on the opposite, single component textures with a maximum ODFintensity of more than 20. However, all steel sheet textures consisting of the classical alpha-, gamma- and/or theta-fibres could satisfactorily be reproduced.

\subsection{Sensitivity Study of a Material Property on Texture}

A major concern in steel sheet industry is to optimise texture in regard to some applications of the sheet. Ideal textures are often known, but the processing conditions to obtain them are not so easily found. The model that we propose may help to answer the following question: "Given a steel sheet of which the processing conditions are known, what are the small changes that one should obtain on the sheet texture in order to improve its performances?". The application of the model will of course not say how the processing conditions should be tuned. However, since the meaning of the parameter model is easy to visualise, we expect that the results of the model application be valuable for people who, in turn, have experience concerning the effect of the processing conditions on texture.

Only the material properties for which we dispose of reliable prediction software can be investigated. Furthermore, the software should use the series expansion coefficients as texture description. The way to proceed consists of

- reproducing the starting experimental texture with the model parameters (Section 2);

- evaluating the material property with the help of the property calculation software for both the experimental and the reproduced texture, and checking that they are similar (see Fig. 10);

- modifying the model parameters one at a time, generating the corresponding textures, and evaluating the material property in those cases;

- combining the parameter modifications that lead to an improvement of the predicted material property and check if the beneficial effects add up. 
-Reproduced experimental texture :

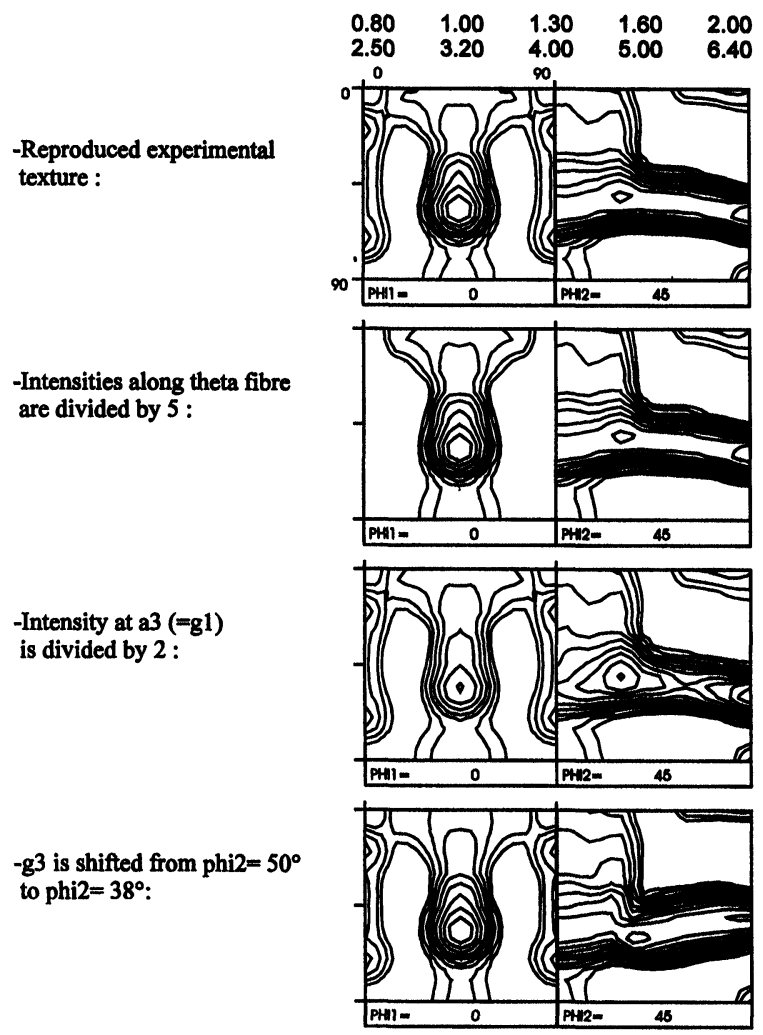

FIGURE 11 Systematic modifications on a reproduced experimental texture by changing the parameter values one at a time.

One should keep in mind that those parameter modifications are only valuable if people concerned with steel sheet production are able to reproduce them. The optimised texture should therefore not be too different from the original one. Figure 11 illustrates how an experimental texture can be modified in a systematic and fully controlled manner. The resulting $R$-value profiles are presented in Fig. 12.

Sensitivity studies based on this model will be the subject of future papers. The first properties that will be investigated are the plastic planar isotropy ( $R$-value profiles) and the formability (FLD diagrams) of steel sheets. 

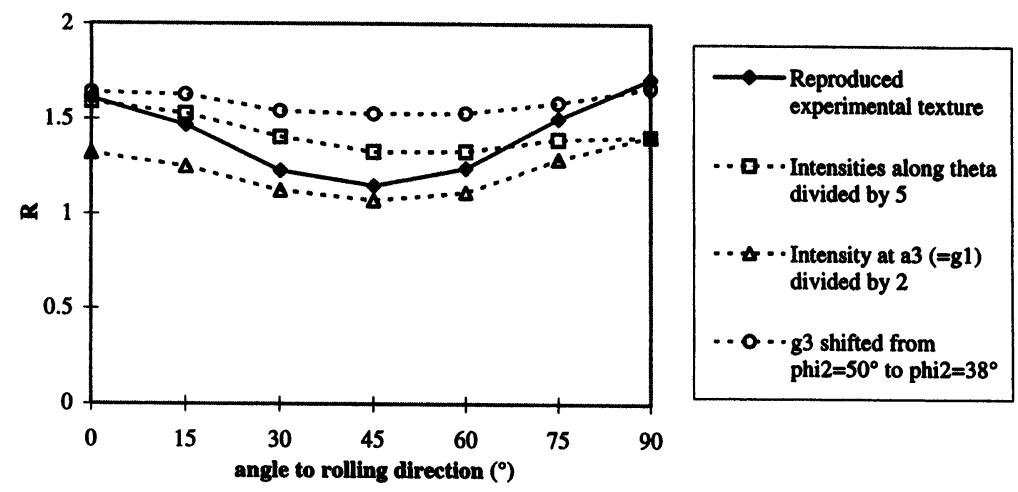

FIGURE $12 R$-value sensitivity due to the parameter value modifications presented in Fig. 11.

\section{CONCLUSIONS}

A new parameter model has been proposed for the characterisation of steel sheet textures. The model proves to be efficient to reproduce all types of steel sheet ODFs consisting of the classical alpha- and gammafibres, and/or the Goss and cube components. The latter two crystallographic components are included in a common theta-fibre which leads to a set of parameters that is easy to comprehend. The originality of the model is to rely on a reduced set of parameters (25) that are directly related to some visual features of the ODF. Determining the parameter values for a given experimental texture is made possible by applying a Fortran routine that requires very little interaction with the user. The resulting parameter values allow an improved three-dimensional visualisation of the ODF. The match between experimental and reproduced textures has been measured quantitatively in the case of four typical steel sheet textures. Some discrepancies were observed between experimental and reproduced ODFs. They are however accepted as such, considering that we only use 25 parameters and that these discrepancies should not play an important role in the prediction of texture dependent material properties. This has been checked for the four reproduced textures, for the study of plastic planar anisotropy. It was finally illustrated how the model can be used for systematic sensitivity studies of texture dependent material properties. Such sensitivity studies will be published in future papers. 


\section{Acknowledgements}

D. Vanderschueren from OCAS n.v. (Zelzate, Belgium) is gratefully acknowledged for fruitful discussions. One of the authors (L.D.) acknowledges his grant as "aspirant" of the "Fonds voor Wetenschappelijk Onderzoek - Vlaanderen". Financial support was also provided by the Federal Government of Belgium (DWTC) through the contract IUAP P4/33.

\section{References}

Altmann, S. (1986). Rotations, Quaternions, and Double Groups, Clarendon Press, Oxford. Bunge, H.J. (1982). Texture Analysis in Material Science, Butterworths, London.

Cai, M.J. and Lee, W.B. (1994). Automatic quantitative analysis of texture components from CODF data, Proc. of ICOTOM-10, Material Science Forum, 157-162, 327-332, Trans Tech Publications, Aedermannsdorf, Switzerland.

Cortie, M.B. (1997). Calculation of texture volume fractions by integration and gaussian fitting, Textures and Microstructures, 29, 155-183.

Dnieprenko, V.N. and Divinski, S.V. (1994). A new approach to describing threedimensional orientation distribution functions in textured materials, Textures and Microstructures, 22, 169-175.

Eschner, Th. (1993). Texture analysis by means of model functions, Textures and Microstructures, 21, 139-146.

Helming, K. (1997). A nearly equal distant grid of orientations for quantitative texture analysis, Textures and Microstructures, 28, 219-230.

Helming, K., Tamm, R. and Fels, B. (1998). An automated component method, Material Science Forum, 273-275, 119-124.

Helming, K. (1998). Texture approximations by model components, Material Science Forum, 273-275, 125-132.

Hirsch, J. and Lücke, K. (1985). Interpretation of the copper-brass texture transition by quantitative ODF analysis, in Preferred Orientation in Deformed Metals and Rocks: an Introduction to Modern Texture Analysis, edited by Wenk H.R., Academic Press, Orlando.

Matthies, S., Vinel, G.W. and Helming, K. (1987). Standard Distributions in Texture Analysis, Vol. I, Akademie-Verslag, Berlin.

Press, W.H., Teukolsky, S.A., Vetterling, W.T. and Flannery, B.P. (1992). Numerical Recipes in Fortran, 2nd. Edition, Cambridge University Press.

Savyolova, T.I. (1993). Approximation of the pole figures and the orientation of distribution of grains in polycrystalline samples by means of canonical normal distributions, Textures and Microstructures, 22, 17-27.

Schouwenaars, R. and Van Houtte, P. (1994). Methods for determining small differences between measured textures, Proc. of ICOTOM-10, Material Science Forum, 157-162, 439-446, Trans Tech Publications, Aedermannsdorf, Switzerland.

Schouwenaars, R., Van Houtte, P., Aernoudt, E., Standaert, C. and Dilewijns, J. (1994). Prediction of the plastic anisotropy of low carbon steel sheet by means of Taylormodelling, ISIJ International, 34, 366-372.

Schouwenaars, R., Van Houtte, P., Van Bael, A., Winters, J. and Mols, K. (1996). Analysis and prediction of the earing behaviour of low carbon steel sheet, Textures and Microstructures, 26-27, 553-570. 
Van Acker, K. (1996). Internal stress states in cold worked metals and in metal matrix composites, Doctoral Thesis, Department MTM, K.U. Leuven.

Van Houtte, P. (1991). A method for the generation of various ghost correction algorithms - the example of the positivity method and the exponential method, Textures and Microstructures, 13, 199-212.

Van Houtte, P. (1995). User Manual of the MTM-FHM software, version 2, Department MTM, K.U. Leuven.

Wagner, F. (1985). Library program, in Quantitative Texture Analysis, Edited by Bunge, H.J. and Esling, C., Deutsche Gesellschaft für Metallkunde. 